\title{
MOTZKIN'S MAXIMAL DENSITY AND RELATED CHROMATIC NUMBERS
}

\author{
Anshika Srivastava - Ram Krishna PandeY* - Om Prakash
}

\begin{abstract}
This paper concerns the problem of determining or estimating the maximal upper density of the sets of nonnegative integers $S$ whose elements do not differ by an element of a given set $M$ of positive integers. We find some exact values and some bounds for the maximal density when the elements of $M$ are generalized Fibonacci numbers of odd order. The generalized Fibonacci sequence of order $r$ is a generalization of the well known Fibonacci sequence, where instead of starting with two predetermined terms, we start with $r$ predetermined terms and each term afterwards is the sum of $r$ preceding terms. We also derive some new properties of the generalized Fibonacci sequence of order $r$. Furthermore, we discuss some related coloring parameters of distance graphs generated by the set $M$.
\end{abstract}

Communicated by Georges Grekos

\section{Introduction}

For a given set $M$ of positive integers, a problem of Motzkin's asks to find the maximal upper density of sets $S$ of nonnegative integers in which no two elements of $S$ are allowed to differ by an element of $M$. Following Motzkin, if $M$ be a given set of positive integers, a set $S$ of nonnegative integers is said to be an $M$-set if $a \in S, b \in S$ implies $a-b \notin M$. Let $S$ be any set of nonnegative integers and $S(x)$ be the number of elements $n \in S$ such that $n \leq x, x \in \mathbb{R}$. We define the upper and lower densities of $S$, denoted respectively by $\bar{\delta}(S)$ and $\underline{\delta}(S)$, by

$$
\bar{\delta}(S)=\limsup _{x \rightarrow \infty} \frac{S(x)}{x}, \quad \underline{\delta}(S)=\liminf _{x \rightarrow \infty} \frac{S(x)}{x} .
$$

2010 Mathematics Subject Classification: 11B05, $11 B 39$.

Keywords: maximal density, generalized Fibonacci numbers, fractional coloring, circular coloring.

* The corresponding author. 


\section{ANSHIKA SRIVASTAVA — RAM KRISHNA PANDEY — OM PRAKASH}

We say that $S$ has density $\delta(S)$, when $\bar{\delta}(S)=\underline{\delta}(S)=\delta(S)$. The parameter of interest is the maximal density of an $M$-set, defined by

$$
\mu(M):=\sup \bar{\delta}(S),
$$

where the supremum is taken over all $M$-sets $S$. Motzkin posed the problem of determining the quantity $\mu(M)$. In 1973, Cantor and Gordon 1 proved that there exists a set $S$ such that $\delta(S)=\mu(M)$, when $M$ is finite. The following two lemmas proved in [1] and [7, respectively, are useful results for bounding $\mu(M)$.

Lemma 1.1. Let $M=\left\{m_{1}, m_{2}, m_{3}, \ldots\right\}$, and $c$ and $m$ be positive integers such that $\operatorname{gcd}(c, m)=1$. Then

$$
\mu(M) \geq \kappa(M):=\sup _{(c, m)=1}(1 / m) \min _{k \geq 1}\left|c m_{k}\right|_{m},
$$

where $|x|_{m}$ denotes the absolute value of the absolutely least remainder of $x$ $(\bmod m)$.

Lemma 1.2. Let $\alpha$ be a real number, $\alpha \in[0,1]$. If for any $M$-set $S$ with $0 \in S$ there exists a positive integer $k$ such that $S(k) \leq(k+1) \alpha$, then $\mu(M) \leq \alpha$.

For a finite set $M$, by a remark of $\mathrm{H}$ a r a la $\mathrm{m}$ b i s [7], we can write $\kappa(M)$ as,

$$
\kappa(M)=\max _{\substack{m=m_{i}+m_{j} \\ 1 \leq k \leq \frac{m}{2}}}(1 / m) \min _{i}\left|k m_{i}\right|_{m},
$$

where $m_{i}, m_{j}$ are distinct elements of $M$.

Motzkin's density problem has wide connections to some coloring problems. The study of Motzkin's density problem is equivalent to the study of the fractional chromatic number of distance graphs. A fractional coloring of a graph $G$ is a mapping $c$ which assigns to each independent set $I$ of $G$ a non-negative weight $c(I)$ such that for each vertex $x, \sum_{x \in I} c(I) \geq 1$. The fractional chromatic number of $G$, denoted by $\chi_{f}(G)$, is the least total weight of a fractional coloring of $G$. Let $D$ be a set of positive integers. The distance graph generated by $D$, denoted by $G(Z, D)$, has set $Z$ as the vertex set, and two vertices $x$ and $y$ are adjacent whenever $|x-y| \in D$. It is proved by $\mathrm{Chang}$ et al. 22 that for any finite set $D$, finding the fractional chromatic number of distance graphs or the maximal density is the same problem. Precisely, they proved the next theorem.

Theorem 1.1. For any finite set $D$ of positive integers, $\mu(D)=1 / \chi_{f}(G(Z, D))$.

Further, the fractional chromatic number is related with another useful chromatic number called the circular chromatic number defined as follows: Let $k \geq 2 d$ be positive integers. A $(k, d)$-coloring of a graph $G$ is a mapping, 


\section{MOTZKIN'S MAXIMAL DENSITY AND RELATED CHROMATIC NUMBERS}

$c: V(G) \rightarrow\{0,1, \ldots k-1\}$, such that $d \leq|c(u)-c(v)| \leq k-d$ for any $u v \in E(G)$. The circular chromatic number of $G$, denoted by $\chi_{c}(G)$, is the minimum ratio $k / d$ such that $G$ admits a $(k, d)$-coloring. It is proved [15] that for any graph $G$,

$$
\chi_{f}(G) \leq \chi_{c}(G) \leq \chi(G)=\left\lceil\chi_{c}(G)\right\rceil,
$$

Moreover, for distance graphs $G(Z, D)$ with distance set $D$, the following theorem [15] relates circular chromatic number with $\kappa(D)$ :

TheOREM 1.2. For any finite set $D$ of positive integers, $\chi_{c}(G(Z, D)) \leq \frac{1}{\kappa(D)}$.

The values and bounds of $\mu(M)$ have been studied for several special families of sets $M([1$, , 2], [5], 6], 7], [4], [8], [9], [13, [12, [11]) but, in general, only for $|M| \leq 2$, complete solution was given by $\mathrm{C}$ ant or and Gordon [1]. In this paper, we intend to study the problem of estimating the maximal density $\mu(U)$ when the set $U$ is finite and consists of the first consecutive generalized Fibonacci numbers of odd order. The Fibonacci sequence has been generalized in many ways. One of them is the Fibonacci sequence $\left\{U_{n}\right\}$ of order $r$. Let $r \geq 1$ be an integer. The Fibonacci sequence $\left\{U_{n}\right\}$ of order $r$ is given by the recurrence relation

$$
U_{n}=U_{n-1}+U_{n-2}+\cdots+U_{n-r}, \quad \text { where } \quad n \geq r
$$

with the $r$ initial terms

$$
U_{n}=0 \quad \text { for } 0 \leq n \leq r-2, \quad \text { and } \quad U_{r-1}=1 .
$$

These generalized Fibonacci numbers are also known as the Fibonacci $r$-step numbers. The usual Fibonacci numbers can be obtained by fixing $r=2$. For small values of $r$, these sequences are sometimes called by individual names. For $r=3$, tribonacci sequence; for $r=4$, tetranacci sequence, and so on. When $r=2$, we know that the sequence $\left(\frac{U_{n}}{U_{n-1}}\right)$ (the ratio of two consecutive Fibonacci numbers) converges to the golden ratio. A fact about this generalization is that, like the usual Fibonacci sequence, $\lim _{n \rightarrow \infty} \frac{U_{n}}{U_{n-1}}$ exists and is the real positive root of the equation $x^{r}-x^{r-1}-\cdots-x-1=0$. All other roots of this equation lie inside the unit circle. The polynomial $x^{r}-x^{r-1}-\cdots-x-1$ has been extensively studied. For detailed work on this polynomial, one may refer to ([3, [10, 14]).

In Section 2, we give some new properties related to $r$-step Fibonacci numbers. These properties are then applied to derive the main results about the maximal density of odd order $r$-step Fibonacci numbers in Section 3 . Further, in Section 4 . we relate our results with the two chromatic numbers, defined earlier, of distance graphs. 


\section{Some properties of the Fibonacci r-step numbers}

In this section, we prove some lemmas concerning the Fibonacci r-step numbers. These lemmas are then applied to prove the results of the next section.

LEMmA 2.1. For $n \geq r+1$, the elements of the set $\left\{U_{n-r-1}, U_{n-1}, U_{n}\right\}$ are in arithmetic progression.

Pr o of. We have,

$$
\begin{aligned}
U_{n} & =U_{n-1}+U_{n-2}+\cdots+U_{n-r} \\
& =U_{n-1}+U_{n-2}+\cdots+U_{n-r}+U_{n-r-1}-U_{n-r-1} \\
& =2 U_{n-1}-U_{n-r-1} .
\end{aligned}
$$

Hence, the lemma.

LEMmA 2.2. Let $r>2$ be an odd integer. Then,

(i) for $r \leq i \leq 2 r-1$,

$$
U_{i}=2^{i-r}=3\left(\sum_{k=1}^{\frac{r-1}{2}} U_{i-2 k}\right)+(-1)^{i+1}
$$

(ii) for $i>2 r-1$,

$$
U_{i}=3\left(\sum_{k=1}^{\frac{r-1}{2}} U_{i-2 k}\right)+\left(\sum_{k=\frac{r+1}{2}}^{r} U_{i-2 k}\right)
$$

Proof.

(i) Clearly, if $i=r$, then $U_{r}=1$, satisfies the formula. So, let $r+1 \leq i \leq 2 r-1$. Using the recurrence $U_{i}=2 U_{i-1}-U_{i-r-1}$ and $U_{r}=U_{r-1}=1$, we have $U_{i}=2^{i-r}$. So, if $i$ is even,

$$
\begin{aligned}
\sum_{k=1}^{\frac{r-1}{2}} U_{i-2 k} & =\left(2^{i-2-r}+2^{i-4-r}+\cdots+2\right)+1 \\
& =2\left(\frac{2^{i-1-r}-1}{3}\right)+1=\frac{2^{i-r}+1}{3},
\end{aligned}
$$

and if $i$ is odd,

$$
\sum_{k=1}^{\frac{r-1}{2}} U_{i-2 k}=\left(2^{i-2-r}+2^{i-4-r}+\cdots+1\right)=\frac{2^{i-r}-1}{3} .
$$

Therefore, for each $i$,

$$
U_{i}=3\left(\sum_{k=1}^{\frac{r-1}{2}} U_{i-2 k}\right)+(-1)^{i+1}
$$


(ii) Since $U_{i}=U_{i-1}+U_{i-2}+\cdots+U_{i-r}$, we have, for $i>2 r-1$,

$$
\begin{aligned}
U_{i}= & \left(U_{i-1}+U_{i-3}+\cdots+U_{i-r+2}\right) \\
& +\left(U_{i-2}+U_{i-4}+v+U_{i-r+1}\right)+U_{i-r} \\
= & \left(2 U_{i-2}-U_{i-r-2}\right)+\cdots+\left(2 U_{i-r+1}-U_{i-2 r+1}\right) \\
& +\left(U_{i-2}+U_{i-4}+\cdots+U_{i-r+1}\right)+U_{i-r} \\
= & 3\left(\sum_{k=1}^{\frac{r-1}{2}} U_{i-2 k}\right)+\left(U_{i-r}-U_{i-r-2}-U_{i-r-4}-\cdots-U_{i-2 r+1}\right) \\
= & 3\left(\sum_{k=1}^{\frac{r-1}{2}} U_{i-2 k}\right)+\left(\sum_{k=\frac{r+1}{2}}^{r} U_{i-2 k}\right) .
\end{aligned}
$$

This completes the proof of the lemma.

Lemma 2.3. Let $r>2$ be an odd integer. Then, for $i \geq r$,

$U_{i}$ and $\left(U_{i-2}+U_{i-4}+\cdots+U_{i-r+1}\right)$ are of opposite parity.

P r o of. We take the following two cases:

Case 1: $(r \leq i \leq 2 r-1)$. Since,

$$
U_{i}=2^{i-r}=3\left(\sum_{k=1}^{\frac{r-1}{2}} U_{i-2 k}\right)+(-1)^{i+1},
$$

we have $U_{i}$ and $\sum_{k=1}^{\frac{r-1}{2}} U_{i-2 k}$ are of opposite parity.

Case 2: $(2 r \geq i)$. We prove this by induction on $i$. The basis step, $i=2 r$ is clearly true as

$$
U_{2 r}=3\left(\sum_{k=1}^{\frac{r-1}{2}} U_{2 r-2 k}\right)+\left(\sum_{k=\frac{r+1}{2}}^{r} U_{2 r-2 k}\right),
$$

where the second sum on the right hand side is equal to 1 .

Now, let the result be true for all $l$ such that $2 r \leq l<i$. Then, we need to prove this for $i$ as well. We have,

$$
\begin{aligned}
\sum_{k=\frac{r+1}{2}}^{r} U_{i-2 k} & =U_{i-r-1}+\left(U_{i-r-3}+U_{i-r-5}+\cdots+U_{i-2 r}\right) \\
& =U_{i-r-1}+\sum_{k=1}^{\frac{r-1}{2}} U_{(i-r-1)-2 k} .
\end{aligned}
$$


By the induction hypothesis, $U_{i-r-1}$ and $\sum_{k=1}^{\frac{r-1}{2}} U_{(i-r-1)-2 k}$ are of opposite parity. This implies that $\sum_{k=\frac{r+1}{2}}^{r} U_{i-2 k}$ is odd. Thus, $U_{i}$ and $\sum_{k=1}^{\frac{r-1}{2}} U_{i-2 k}$ are of opposite parity by Lemma 2.2. Hence, the lemma.

We use the following elementary property of positive integers in the proof of the next lemma.

REMARK 2.1. Let $a, b, c$, and $d$ be positive integers with $b>d$, and $\frac{a}{b}<\frac{c}{d}$. Then

$$
\frac{a-c}{b-d}<\frac{a}{b}<\frac{c}{d} .
$$

Lemma 2.4. Let $r>2$ be an odd integer and $i \geq 2 r-1$. Then,

$$
\frac{2^{r-3}-1}{3\left(2^{r-3}\right)}<\frac{U_{i-2}+U_{i-4}+\cdots+U_{i-r+1}}{U_{i}}<\frac{2^{r-2}+1}{3\left(2^{r-2}\right)} .
$$

Further, for $n \geq 2 r+1$, the sequence $(f(n))$, where

$$
f(n)=\frac{U_{n-2}+U_{n-4}+\cdots+U_{n-r+1}+\frac{2^{r-2}+1}{3}}{U_{n}+2^{r-2}},
$$

is strictly decreasing.

P r o of. Since $U_{n}=2 U_{n-1}-U_{n-r-1}$ for $n \geq r+1$, we have for $n \geq 2 r, \frac{U_{n}}{U_{n-1}}<2$.

Hence, $\frac{U_{n}}{U_{n-i}}<2^{i}$, if $n \geq r+i$. This implies that

$$
\frac{U_{i-2}+U_{i-4}+\cdots+U_{i-r+1}}{U_{i}}>\frac{1}{2^{2}}+\frac{1}{2^{4}}+\cdots+\frac{1}{2^{r-1}},
$$

if $i \geq 2 r-1$. Thus, if $i \geq 2 r-1$, then

$$
\frac{U_{i-2}+U_{i-4}+\cdots+U_{i-r+1}}{U_{i}}>\frac{1}{2^{2}}+\frac{1}{2^{4}}+\cdots+\frac{1}{2^{r-1}}>\frac{2^{r-3}-1}{3\left(2^{r-3}\right)} .
$$

On the other hand, for $i \geq 2 r-1$,

$$
U_{i}>3\left(\sum_{k=1}^{\frac{r-1}{2}} U_{i-2 k}\right),
$$

which gives

$$
\frac{U_{i-2}+U_{i-4}+\cdots+U_{i-r+1}}{U_{i}}<\frac{2^{r-2}+1}{3\left(2^{r-2}\right)} .
$$

This proves the first part of the lemma. 
For the second part, we apply induction on $n$ to show that $f(n)<f(n-1)$ for all $n \geq 2 r+2$. Notice that $f(2 r+2)=\frac{1}{3}-\frac{2}{3\left(U_{2 r+2}+2^{r-2}\right)}$, and $f(2 r+1)=\frac{1}{3}$. Hence, the basis step is satisfied. Since $U_{n}=2 U_{n-1}-U_{n-r-1}$, we have

$$
\begin{aligned}
f(n) & =\frac{U_{n-2}+U_{n-4}+\cdots+U_{n-r+1}+\frac{2^{r-2}+1}{3}}{U_{n}+2^{r-2}} \\
& =\frac{2\left(\sum_{k=1}^{\frac{r-1}{2}} U_{n-1-2 k}\right)-\left(\sum_{k=1}^{\frac{r-1}{2}} U_{n-r-1-2 k}\right)+\frac{2^{r-2}+1}{3}}{2 U_{n-1}-U_{n-r-1}+2^{r-2}} \\
& =\frac{2\left(\sum_{k=1}^{\frac{r-1}{2}} U_{n-1-2 k}+\frac{2^{r-2}+1}{3}\right)-\left(\sum_{k=1}^{\frac{r-1}{2}} U_{n-r-1-2 k}+\frac{2^{r-2}+1}{3}\right)}{2\left(U_{n-1}+2^{r-2}\right)-\left(U_{n-r-1}+2^{r-2}\right)} .
\end{aligned}
$$

Now, by induction hypothesis assume that

$$
\begin{aligned}
f(n-1)= & \frac{\sum_{k=1}^{\frac{r-1}{2}} U_{n-1-2 k}+\frac{2^{r-2}+1}{3}}{\left(U_{n-1}+2^{r-2}\right)}<f(n-2)<\cdots f(n-r-1) \\
= & \frac{\sum_{k=1}^{\frac{r-1}{2}} U_{n-r-1-2 k}+\frac{2^{r-2}+1}{3}}{\left(U_{n-r-1}+2^{r-2}\right)}<\cdots f(2 r+1) .
\end{aligned}
$$

Letting

$$
\begin{array}{ll}
a=2\left(\sum_{k=1}^{\frac{r-1}{2}} U_{n-1-2 k}+\frac{2^{r-2}+1}{3}\right), & b=2\left(U_{n-1}+2^{r-2}\right), \\
c=\sum_{k=1}^{\frac{r-1}{2}} U_{n-r-1-2 k}+\frac{2^{r-2}+1}{3}, & \text { and } \quad d=U_{n-r-1}+2^{r-2}
\end{array}
$$

in Remark (2.1), we have

$$
\begin{gathered}
\frac{2\left(\sum_{k=1}^{\frac{r-1}{2}} U_{n-1-2 k}+\frac{2^{r-2}+1}{3}\right)-\left(\sum_{k=1}^{\frac{r-1}{2}} U_{n-r-1-2 k}+\frac{2^{r-2}+1}{3}\right)}{2\left(U_{n-1}+2^{r-2}\right)-\left(U_{n-r-1}+2^{r-2}\right)} \\
<\frac{\sum_{k=1}^{\frac{r-1}{2}} U_{n-1-2 k}+\frac{2^{r-2}+1}{3}}{\left(U_{n-1}+2^{r-2}\right)}<\frac{\sum_{k=1}^{\frac{r-1}{2}} U_{n-r-1-2 k}+\frac{2^{r-2}+1}{3}}{\left(U_{n-r-1}+2^{r-2}\right)} .
\end{gathered}
$$


This gives, $f(n)<f(n-1)$. Thus, $f(n)<f(n-1)$ for all $n \geq 2 r+2$.

Hence, the lemma.

REMARK 2.2. We have for $r \leq n \leq 2 r-1$,

$$
U_{n}=2^{n-r}=3\left(\sum_{k=1}^{\frac{r-1}{2}} U_{n-2 k}\right)+(-1)^{n+1} .
$$

Therefore, if $n$ is odd, then $f(n)=\frac{1}{3}$, and if $n$ is even, then $f(n)=\frac{1}{3}+\frac{2}{3\left(U_{n}+2^{r-2}\right)}$. Further, $f(2 r)=\frac{1}{3}$. Thus, the finite sequence $(f(n))_{n=r}^{2 r}$ is not monotonic.

\section{Main Results}

TheOREM 3.1. Let $r>2$ be an odd integer and let $U=\left\{U_{r}, U_{r+1}, \ldots, U_{n}\right\}$. Then,

(i) if $r+1 \leq n \leq 2 r+1$, then

(ii) if $n>2 r+1$, then

$$
\mu(U)=\kappa(U)=\frac{1}{3}
$$

$$
\frac{1}{3}>\mu(U) \geq \kappa(U) \geq \frac{\left(U_{n-2}+U_{n-4}+\cdots+U_{n-r+1}+1\right) 2^{r-3}-U_{n}\left(\frac{2^{r-3}-1}{3}\right)}{U_{n}+2^{r-2}} .
$$

Pr o of. (i) We have, for $r \leq i \leq 2 r-1$,

In addition, we also have

$$
U_{i}=2^{i-r} \equiv \pm 1 \quad(\bmod 3)
$$

$$
\begin{array}{ll}
U_{2 r}=2 U_{2 r-1}-U_{r-1}=2^{r}-1 \equiv 1 & (\bmod 3), \\
U_{2 r+1}=2 U_{2 r}-U_{r}=2^{r+1}-3 \equiv 1 & (\bmod 3) .
\end{array}
$$

Therefore, taking $c=1$ and $m=3$ in Lemma 1.1, we have $\mu(U) \geq \kappa(U) \geq \frac{1}{3}$. On the other hand, any $U$-set cannot contain any consecutive integers as well as consecutive integers of same parity as $\{1,2\} \subseteq U$. This implies that, $\mu(U) \leq \frac{1}{3}$. This completes the proof in this case.

(ii) Since $U_{n}$ and $\left(U_{n-2}+U_{n-4}+\cdots+U_{n-r+1}\right)$ are of opposite parity and $\frac{2^{r-2}+1}{3}$ is an odd integer, so

$$
x=\frac{\left(U_{n}+2^{r-2}\right)-\left(U_{n-2}+U_{n-4}+\cdots+U_{n-r+1}+\frac{2^{r-2}+1}{3}\right)}{2}
$$

is an integer. 
We claim that for $r \leq i \leq n$,

$$
\begin{aligned}
& U_{i} x \equiv \frac{U_{n}+2^{r-2}}{2} \\
& -\frac{U_{i}\left(\sum_{k=1}^{\frac{r-1}{2}} U_{n-2 k}+\frac{2^{r-2}+1}{3}\right)-\left(\sum_{k=1}^{\frac{r-1}{2}} U_{i-2 k}\right)\left(U_{n}+2^{r-2}\right)}{2}\left(\bmod U_{n}+2^{r-2}\right) .
\end{aligned}
$$

If $U_{i}$ is even, then

$$
\begin{aligned}
U_{i} x & =U_{i} \frac{\left(U_{n}+2^{r-2}\right)-\left(U_{n-2}+U_{n-4}+\cdots+U_{n-r+1}+\frac{2^{r-2}+1}{3}\right)}{2} \\
& \equiv-U_{i} \frac{\left(U_{n-2}+U_{n-4}+\cdots+U_{n-r+1}+\frac{2^{r-2}+1}{3}\right)}{2} \\
& \equiv \frac{\left(U_{i-2}+\cdots+U_{i-r+1}+1\right)\left(U_{n}+2^{r-2}\right)}{2} \\
& -U_{i} \frac{\left(U_{n-2}+\cdots+U_{n-r+1}+\frac{2^{r-2}+1}{3}\right)}{2}\left(\bmod U_{n}+2^{r-2}\right) .
\end{aligned}
$$

Next, if $U_{i}$ is odd, then

$$
\begin{aligned}
U_{i} x= & U_{i} \frac{\left(U_{n}+2^{r-2}\right)-\left(U_{n-2}+U_{n-4}+\cdots+U_{n-r+1}+\frac{2^{r-2}+1}{3}\right)}{2} \\
\equiv & \frac{U_{n}+2^{r-2}}{2}-U_{i} \frac{\left(U_{n-2}+U_{n-4}+\cdots+U_{n-r+1}+\frac{2^{r-2}+1}{3}\right)}{2} \\
\equiv & \frac{\left(U_{i-2}+\cdots+U_{i-r+1}+1\right)\left(U_{n}+2^{r-2}\right)}{2} \\
& -U_{i} \frac{\left(U_{n-2}+\cdots+U_{n-r+1}+\frac{2^{r-2}+1}{3}\right)}{2}\left(\bmod U_{n}+2^{r-2}\right) .
\end{aligned}
$$

Thus, we obtain our claim.

Next, we have for $r \leq i \leq 2 r-1$,

$$
U_{i}=3\left(\sum_{k=1}^{\frac{r-1}{2}} U_{i-2 k}\right)+(-1)^{i+1} .
$$

Hence, it is easy to see that

$$
\left(U_{i-2}+\cdots+U_{i-r+1}+1\right)\left(U_{n}+2^{r-2}\right)-U_{i}\left(U_{n-2}+\cdots+U_{n-r+1}+\frac{2^{r-2}+1}{3}\right)>0 .
$$




\section{ANSHIKA SRIVASTAVA — RAM KRISHNA PANDEY — OM PRAKASH}

Further, for $n \geq i>2 r-1$, we have, by Lemma 2.4, that

This gives

$$
\frac{2^{r-3}-1}{3\left(2^{r-3}\right)}<\frac{U_{i-2}+U_{i-4}+\cdots+U_{i-r+1}}{U_{i}} \text {. }
$$

Therefore,

$$
\frac{2^{r-3}-1}{3\left(2^{r-3}\right)}<\frac{U_{i-2}+U_{i-4}+\cdots+U_{i-r+1}+1}{U_{i}} .
$$

$$
\begin{aligned}
\frac{2^{r-3}-1}{3\left(2^{r-3}\right)} & <\frac{U_{i-2}+U_{i-4}+\cdots+U_{i-r+1}+\frac{2^{r-2}+1}{3}}{U_{i}+2^{r-2}} \\
& <\frac{U_{i-2}+U_{i-4}+\cdots+U_{i-r+1}+1}{U_{i}}
\end{aligned}
$$

Thus,

$\left(U_{i-2}+\cdots+U_{i-r+1}+1\right)\left(U_{n}+2^{r-2}\right)-U_{i}\left(U_{n-2}+\cdots+U_{n-r+1}+\frac{2^{r-2}+1}{3}\right)>0$.

Now for all $i$, there are two cases: either

$$
U_{i}\left(\sum_{k=1}^{\frac{r-1}{2}} U_{n-2 k}+\frac{2^{r-2}+1}{3}\right)>\left(\sum_{k=1}^{\frac{r-1}{2}} U_{i-2 k}\right)\left(U_{n}+2^{r-2}\right)
$$

or

$$
U_{i}\left(\sum_{k=1}^{\frac{r-1}{2}} U_{n-2 k}+\frac{2^{r-2}+1}{3}\right)<\left(\sum_{k=1}^{\frac{r-1}{2}} U_{i-2 k}\right)\left(U_{n}+2^{r-2}\right) .
$$

Equality may also hold for some $i$, but in that case the maximum absolute remainder modulo $\left(U_{n}+2^{r-2}\right)$ is $\frac{U_{n}+2^{r-2}}{2}$, which is not helpful in calculating $\kappa(M)$ (see (1.1)) as we shall see below in both the cases that inequality does hold for some $i$.

CASE 1. $U_{i}\left(\sum_{k=1}^{\frac{r-1}{2}} U_{n-2 k}+\frac{2^{r-2}+1}{3}\right)>\left(\sum_{k=1}^{\frac{r-1}{2}} U_{i-2 k}\right)\left(U_{n}+2^{r-2}\right)$.

Clearly,

$$
\begin{aligned}
& \left|U_{i} x\right|_{\left(U_{n}+2^{r-2}\right)} \\
& =\frac{U_{n}+2^{r-2}}{2}-\frac{U_{i}\left(\sum_{k=1}^{\frac{r-1}{2}} U_{n-2 k}+\frac{2^{r-2}+1}{3}\right)-\left(\sum_{k=1}^{\frac{r-1}{2}} U_{i-2 k}\right)\left(U_{n}+2^{r-2}\right)}{2} .
\end{aligned}
$$


In addition, by Lemma 2.4, for all $i, n \geq i \geq 2 r-1$, we have

$$
\frac{\sum_{k=1}^{\frac{r-1}{2}} U_{n-2 k}+\frac{2^{r-2}+1}{3}}{U_{n}+2^{r-2}} \leq \frac{\sum_{k=1}^{\frac{r-1}{2}} U_{i-2 k}+\frac{2^{r-2}+1}{3}}{U_{i}+2^{r-2}} .
$$

Therefore,

$$
\begin{aligned}
0 & <U_{i}\left(\sum_{k=1}^{\frac{r-1}{2}} U_{n-2 k}+\frac{2^{r-2}+1}{3}\right)-\left(\sum_{k=1}^{\frac{r-1}{2}} U_{i-2 k}\right)\left(U_{n}+2^{r-2}\right) \\
& \leq\left(U_{n}+2^{r-2}\right)\left(\frac{2^{r-2}+1}{3}\right)-2^{r-2}\left(\sum_{k=1}^{\frac{r-1}{2}} U_{n-2 k}+\frac{2^{r-2}+1}{3}\right) \\
& =U_{n}\left(\frac{2^{r-2}+1}{3}\right)-2^{r-2}\left(\sum_{k=1}^{\frac{r-1}{2}} U_{n-2 k}\right) .
\end{aligned}
$$

Observe here that equality holds when $i=n$, Therefore, for all $i, n \geq i \geq 2 r-1$, we have

$$
\begin{aligned}
& \min \left(\left|U_{i} x\right|\right)_{\left(U_{n}+2^{r-2}\right)}=\frac{U_{n}+2^{r-2}}{2}-\frac{U_{n}\left(\frac{2^{r-2}+1}{3}\right)-2^{r-2}\left(\sum_{k=1}^{\frac{r-1}{2}} U_{n-2 k}\right)}{2} \\
& =\left(U_{n-2}+U_{n-4}+\cdots+U_{n-r+1}+1\right) 2^{r-3}-\frac{U_{n}}{2}\left(\frac{2^{r-2}-2}{3}\right) \\
& =\left(U_{n-2}+U_{n-4}+\cdots+U_{n-r+1}+1\right) 2^{r-3}-U_{n}\left(\frac{2^{r-3}-1}{3}\right) \text {. }
\end{aligned}
$$

Now, let $2 r-2 \geq i \geq r$. We observe below that the inequality condition of Case 1 is satisfied by only those $U_{i}$ for which $i$ is odd (the rest of the $U_{i}$ s will satisfy the reverse inequality condition mentioned ahead in Case 2).

Let $i$ be odd such that $r \leq i \leq 2 r-2$. Clearly,

$$
U_{i}=3\left(\sum_{k=1}^{\frac{r-1}{2}} U_{i-2 k}\right)+1
$$

and, as $n>2 r+1>2 r-1$, we have

$$
\frac{2^{r-3}-1}{3\left(2^{r-3}\right)}<\frac{\sum_{k=1}^{\frac{r-1}{2}} U_{n-2 k}}{U_{n}} \text {. }
$$


This implies

$$
\frac{U_{n}-3 \sum_{k=1}^{\frac{r-1}{2}} U_{n-2 k}-1}{U_{n}+2^{r-2}}<\frac{1}{2^{r-3}} \leq \frac{1}{U_{i}}=\frac{U_{i}-3 \sum_{k=1}^{\frac{r-1}{2}} U_{i-2 k}}{U_{i}}
$$

Therefore,

$$
U_{i}\left(\sum_{k=1}^{\frac{r-1}{2}} U_{n-2 k}+\frac{2^{r-2}+1}{3}\right)>\left(\sum_{k=1}^{\frac{r-1}{2}} U_{i-2 k}\right)\left(U_{n}+2^{r-2}\right) .
$$

Observe that, since $n>2 r+1$, we have $U_{n}>3 \sum_{k=1}^{\frac{r-1}{2}} U_{n-2 k}+1$. Therefore,

$$
\begin{aligned}
& U_{n}\left(\frac{2^{r-2}+1}{3}\right)-2^{r-2}\left(\sum_{k=1}^{\frac{r-1}{2}} U_{n-2 k}\right) \\
& \quad-\left(U_{i}\left(\sum_{k=1}^{\frac{r-1}{2}} U_{n-2 k}+\frac{2^{r-2}+1}{3}\right)-\left(\sum_{k=1}^{\frac{r-1}{2}} U_{i-2 k}\right)\left(U_{n}+2^{r-2}\right)\right) \\
& =\frac{U_{i}+2^{r-2}}{3}\left(U_{n}-3 \sum_{k=1}^{\frac{r-1}{2}} U_{n-2 k}-1\right) \geq 0 .
\end{aligned}
$$

Therefore, in this case, for all $i, r \leq i \leq n$, $\min \left(\left|U_{i} x\right|\right)_{\left(U_{n}+2^{r-2}\right)}=\left(U_{n-2}+U_{n-4}+\cdots+U_{n-r+1}+1\right) 2^{r-3}-U_{n}\left(\frac{2^{r-3}-1}{3}\right)$.

CASE 2. $U_{i}\left(\sum_{k=1}^{\frac{r-1}{2}} U_{n-2 k}+\frac{2^{r-2}+1}{3}\right)<\left(\sum_{k=1}^{\frac{r-1}{2}} U_{i-2 k}\right)\left(U_{n}+2^{r-2}\right)$.

We have,

$$
\begin{aligned}
& U_{i}\left(\sum_{k=1}^{\frac{r-1}{2}} U_{n-2 k}+\frac{2^{r-2}+1}{3}\right)-\left(\sum_{k=1}^{\frac{r-1}{2}} U_{i-2 k}\right)\left(U_{n}+2^{r-2}\right) \\
& =U_{i}\left(\sum_{k=1}^{\frac{r-1}{2}} U_{n-2 k}\right)-\left(\sum_{k=1}^{\frac{r-1}{2}} U_{i-2 k}\right) U_{n}+\left(U_{i} \frac{2^{r-2}+1}{3}-2^{r-2}\left(\sum_{k=1}^{\frac{r-1}{2}} U_{i-2 k}\right)\right)
\end{aligned}
$$


Therefore, for all $i, n \geq i \geq 2 r-1$, we have

$$
\begin{aligned}
& U_{i}\left(\sum_{k=1}^{\frac{r-1}{2}} U_{n-2 k}+\frac{2^{r-2}+1}{3}\right)-\left(\sum_{k=1}^{\frac{r-1}{2}} U_{i-2 k}\right)\left(U_{n}+2^{r-2}\right) \\
& \geq U_{i}\left(\sum_{k=1}^{\frac{r-1}{2}} U_{n-2 k}\right)-\left(\sum_{k=1}^{\frac{r-1}{2}} U_{i-2 k}\right) U_{n} \\
& \geq 2^{r-2} \frac{3}{2^{r-2}+1}\left(\sum_{k=1}^{\frac{r-1}{2}} U_{i-2 k}\right)\left(\sum_{k=1}^{\frac{r-1}{2}} U_{n-2 k}\right)-\left(\sum_{k=1}^{\frac{r-1}{2}} U_{i-2 k}\right) U_{n} \\
& \\
& =\frac{3\left(\sum_{k=1}^{\frac{r-1}{2}} U_{i-2 k}\right)}{2^{r-2}+1}\left(2^{r-2}\left(\sum_{k=1}^{\frac{r-1}{2}} U_{n-2 k}\right)-U_{n}\left(\frac{2^{r-2}+1}{3}\right)\right) \\
& \geq 2^{r-2}\left(\sum_{k=1}^{\frac{r-1}{2}} U_{n-2 k}\right)-U_{n}\left(\frac{2^{r-2}+1}{3}\right) .
\end{aligned}
$$

Since, for $n>2 r+1>2 r-1$, we have

$$
\frac{2^{r-3}-1}{3\left(2^{r-3}\right)}<\frac{\sum_{k=1}^{\frac{r-1}{2}} U_{n-2 k}}{U_{n}} .
$$

This implies that

$$
U_{n}\left(\frac{2^{r-2}+1}{3}\right)-2^{r-2} \sum_{k=1}^{\frac{r-1}{2}} U_{n-2 k}<U_{n}+2^{r-2} .
$$

Therefore, for all $i, n \geq i \geq 2 r-1$, we have

$$
\begin{aligned}
& \left|U_{i} x\right|_{U_{n}+2^{r-2}} \\
& =\frac{U_{n}+2^{r-2}}{2}+\frac{U_{i}\left(\sum_{k=1}^{\frac{r-1}{2}} U_{n-2 k}+\frac{2^{r-2}+1}{3}\right)-\left(\sum_{k=1}^{\frac{r-1}{2}} U_{i-2 k}\right)\left(U_{n}+2^{r-2}\right)}{2} .
\end{aligned}
$$

Observe that for $n>2 r+1$, we have $U_{n}>3 \sum_{k=1}^{\frac{r-1}{2}} U_{n-2 k}+1$, which implies

$$
\frac{3 \sum_{k=1}^{\frac{r-1}{2}} U_{n-2 k}+1-U_{n}}{U_{n}+2^{r-2}}<\frac{1}{2^{r-2}}
$$


Now, let $2 r-2 \geq i \geq r$ and $i$ is even. Clearly,

$$
U_{i}=3\left(\sum_{k=1}^{\frac{r-1}{2}} U_{i-2 k}\right)-1
$$

Therefore,

$$
\frac{3 \sum_{k=1}^{\frac{r-1}{2}} U_{n-2 k}+1-U_{n}}{U_{n}+2^{r-2}}<\frac{3 \sum_{k=1}^{\frac{r-1}{2}} U_{i-2 k}-U_{i}}{U_{i}}
$$

and hence

$$
U_{i}\left(\sum_{k=1}^{\frac{r-1}{2}} U_{n-2 k}+\frac{2^{r-2}+1}{3}\right)-\left(\sum_{k=1}^{\frac{r-1}{2}} U_{i-2 k}\right)\left(U_{n}+2^{r-2}\right)<0 .
$$

Moreover, when $U_{i}=3\left(\sum_{k=1}^{\frac{r-1}{2}} U_{i-2 k}\right)-1$,

$$
\begin{aligned}
& U_{i}\left(\sum_{k=1}^{\frac{r-1}{2}} U_{n-2 k}+\frac{2^{r-2}+1}{3}\right)-\left(\sum_{k=1}^{\frac{r-1}{2}} U_{i-2 k}\right)\left(U_{n}+2^{r-2}\right) \\
& \quad-\left(2^{r-2}\left(\sum_{k=1}^{\frac{r-1}{2}} U_{n-2 k}\right)-U_{n}\left(\frac{2^{r-2}+1}{3}\right)\right) \\
& =\frac{U_{i}-2^{r-2}}{3}\left(3 \sum_{k=1}^{\frac{r-1}{2}} U_{n-2 k}+1-U_{n}\right) \leq 0 .
\end{aligned}
$$

Notice that the above inequality is sharp for $i=2 r-2$. Therefore, in this case for all $\mathrm{i}, r \leq i \leq n$,

$$
\begin{aligned}
\min \left(\left|U_{i} x\right|\right)_{\left(U_{n}+2^{r-2}\right)} & =\frac{U_{n}+2^{r-2}}{2}+\frac{2^{r-2}\left(\sum_{k=1}^{\frac{r-1}{2}} U_{n-2 k}\right)-U_{n}\left(\frac{2^{r-2}+1}{3}\right)}{2} \\
& =\left(U_{n-2}+U_{n-4}+\cdots+{ }_{n-r+1}+1\right) 2^{r-3}-U_{n}\left(\frac{2^{r-3}-1}{3}\right) .
\end{aligned}
$$

Therefore, by the definition of $\kappa(U)$ (see (1.1)), in both the cases,

$$
\mu(U) \geq \kappa(U) \geq \frac{\left(U_{n-2}+U_{n-4}+\cdots+U_{n-r+1}+1\right) 2^{r-3}-U_{n}\left(\frac{2^{r-3}-1}{3}\right)}{U_{n}+2^{r-2}} .
$$

On the other hand, when $r$ is odd,

$$
U_{2 r+2}=2 U_{2 r+1}-U_{r+1}=2^{r+2}-8 \equiv 0 \quad(\bmod 3) .
$$


We decompose $\left\{0,1,2, \ldots, U_{2 r+2}\right\}$ into the sets $\{3 i, 3 i+1,3 i+2\}$ and $\left\{U_{2 r+2}\right\}$, where $0 \leq i \leq \frac{U_{2 r+2}-3}{3}$. Let $S$ be an $U$-set with $0 \in S$. Then it is clear that $|S \cap\{3 i, 3 i+1,3 i+2\}| \leq 1$ and $U_{2 r+2} \notin S$. Thus, using Lemma 1.2, for $n>2 r+1$, we have $\mu(U) \leq \mu\left\{U_{r}, U_{r+1}, \ldots, U_{2 r+2}\right\} \leq \frac{U_{2 r+2}}{3\left(U_{2 r+2}+1\right)}<\frac{1}{3}$. Therefore,

$$
\frac{1}{3}>\mu(U) \geq \kappa(U) \geq \frac{\left(U_{n-2}+U_{n-4}+\cdots+U_{n-r+1}+1\right) 2^{r-3}-U_{n}\left(\frac{2^{r-3}-1}{3}\right)}{U_{n}+2^{r-2}} .
$$

This completes the proof of the theorem.

The following theorem directly follows from the above theorem.

TheORem 3.2. Let $U=\left\{U_{r}, U_{r+1}, \ldots, U_{n}\right\}$ and $n>2 r+1$. Then

$$
\kappa(U) \geq \frac{1}{3}-2^{r-3}\left(\frac{1}{3}-\frac{\alpha-1}{\alpha+1}\right)>\frac{1}{4},
$$

where $\alpha$ is the real root of the polynomial $f(x)=x^{r}-x^{r-1}-x^{r-2}-\cdots-x-1$.

P r o o f. We have that $\left(\frac{U_{n-2}+U_{n-4}+\cdots+U_{n-r+1}+\frac{2^{r-2}+1}{3}}{U_{n}+2^{r-2}}\right)$ is a decreasing sequence, by Lemma 2.4. Now,

$$
\begin{aligned}
& \frac{\left(U_{n-2}+U_{n-4}+\cdots+U_{n-r+1}+\frac{2^{r-2}+1}{3}\right) 2^{r-3}-\left(U_{n}+2^{r-2}\right) \frac{2^{r-3}-1}{3}}{U_{n}+2^{r-2}} \\
& =\frac{\left(U_{n-2}+U_{n-4}+\cdots+U_{n-r+1}+1\right) 2^{r-3}-U_{n}\left(\frac{2^{r-3}-1}{3}\right)}{U_{n}+2^{r-2}} \\
& =z_{n}, \quad \text { say. }
\end{aligned}
$$

Then $\left(z_{n}\right)$ is also a decreasing sequence. We find the limit of the sequence $\left(z_{n}\right)$. Note that $\left(U_{n}\right)$ is an increasing sequence. Hence, $U_{n} \rightarrow \infty$ as $n \rightarrow \infty$. Let

$$
\begin{aligned}
z & =\lim _{n \rightarrow \infty} z_{n} \\
& =\lim _{n \rightarrow \infty} \frac{\left(U_{n-2}+U_{n-4}+\cdots+U_{n-r+1}+1\right) 2^{r-3}-U_{n}\left(\frac{2^{r-3}-1}{3}\right)}{U_{n}+2^{r-2}} \\
& =\lim _{n \rightarrow \infty} \frac{\left(\frac{U_{n-2}}{U_{n}}+\frac{U_{n-4}}{U_{n}}+\cdots+\frac{U_{n-r+1}}{U_{n}}+\frac{1}{U_{n}}\right) 2^{r-3}-\left(\frac{2^{r-3}-1}{3}\right)}{1+\frac{2^{r-2}}{U_{n}}} .
\end{aligned}
$$

Since

$\lim _{n \rightarrow \infty} \frac{U_{n}}{U_{n-1}}=\alpha$, we have $\lim _{n \rightarrow \infty} \frac{U_{n}}{U_{n-k}}=\lim _{n \rightarrow \infty} \frac{U_{n}}{U_{n-1}} \cdot \frac{U_{n-1}}{U_{n-2}} \cdots \frac{U_{n-k+1}}{U_{n-k}}=\alpha^{k}$. 
Therefore,

$$
z=\left(\frac{1}{\alpha^{2}}+\frac{1}{\alpha^{4}}+\cdots+\frac{1}{\alpha^{r-1}}\right) 2^{r-3}-\left(\frac{2^{r-3}-1}{3}\right) .
$$

Letting $\alpha$ the real positive root of $f(x)$, we get

$$
\begin{aligned}
\alpha^{r}-\alpha^{r-1}-\alpha^{r-2}-\cdots \alpha-1 & =0 \\
\Rightarrow 1-\frac{1}{\alpha}-\frac{1}{\alpha^{2}}-\cdots-\frac{1}{\alpha^{r}} & =0 \\
\Rightarrow 1-\frac{1}{\alpha}-\frac{1}{\alpha^{3}}-\cdots-\frac{1}{\alpha^{r}} & =\frac{1}{\alpha^{2}}+\frac{1}{\alpha^{4}}+\cdots+\frac{1}{\alpha^{r-1}} \\
\Rightarrow 1-\frac{1}{\alpha}-\frac{1}{\alpha}\left(\frac{1}{\alpha^{2}}+\frac{1}{\alpha^{4}}+\cdots+\frac{1}{\alpha^{r-1}}\right) & =\frac{1}{\alpha^{2}}+\frac{1}{\alpha^{4}}+\cdots+\frac{1}{\alpha^{r-1}} \\
\Rightarrow \frac{1}{\alpha^{2}}+\frac{1}{\alpha^{4}}+\cdots+\frac{1}{\alpha^{r-1}} & =\frac{\alpha-1}{\alpha+1} .
\end{aligned}
$$

Therefore,

$$
z=\left(\frac{\alpha-1}{\alpha+1}\right) 2^{r-3}-\left(\frac{2^{r-3}-1}{3}\right)=\frac{1}{3}-2^{r-3}\left(\frac{1}{3}-\frac{\alpha-1}{\alpha+1}\right) .
$$

However, $z$ is the limit of the decreasing sequence $\left(z_{n}\right)$, where

$$
z_{n}=\frac{\left(U_{n-2}+U_{n-4}+\cdots+U_{n-r+1}+1\right) 2^{r-3}-U_{n}\left(\frac{2^{r-3}-1}{3}\right)}{U_{n}+2^{r-2}} .
$$

This implies that for every $n, z_{n} \geq z$. Now using Theorem 3.1, we have

$$
\begin{aligned}
\kappa(U) & \geq \frac{\left(U_{n-2}+U_{n-4}+\cdots+U_{n-r+1}+1\right) 2^{r-3}-U_{n}\left(\frac{2^{r-3}-1}{3}\right)}{U_{n}+2^{r-2}} \\
& \geq \frac{1}{3}-2^{r-3}\left(\frac{1}{3}-\frac{\alpha-1}{\alpha+1}\right) .
\end{aligned}
$$

Let $g(x)=(x-1) f(x)=x^{r+1}-2 x^{r}+1$. Since $\frac{2^{r}+1}{3}<2^{r-1}$, we have

$$
\begin{aligned}
g\left(2-\frac{3}{2^{r}+1}\right) & =\left(2-\frac{3}{2^{r}+1}\right)^{r+1}-2\left(2-\frac{3}{2^{r}+1}\right)^{r}+1 \\
& <\left(2-\frac{1}{2^{r-1}}\right)^{r+1}-2\left(2-\frac{1}{2^{r-1}}\right)^{r}+1 \\
& =-2\left(1-\frac{1}{2^{r}}\right)^{r}+1<0 .
\end{aligned}
$$


Now as $g(2)=1>0, g(x)$ has at least one root in the interval $\left(2-\frac{3}{2^{r}+1}, 2\right)$. But using Descartes' rule of signs, $g(x)$ has only two positive roots. Therefore, positive root of $g(x)$ other that 1 is $\alpha$. Hence, $\alpha>2-\frac{3}{2^{r}+1}=\frac{2^{r-1}-1}{2^{r}+1}$, which implies that $\frac{\alpha-1}{\alpha+1}>\frac{2^{r-1}-1}{32^{r-1}}$. Therefore,

$$
\kappa(U) \geq \frac{1}{3}-2^{r-3}\left(\frac{1}{3}-\frac{\alpha-1}{\alpha+1}\right)>\frac{1}{4} .
$$

This completes the proof of the theorem.

\section{Chromatic number of the distance graph $G(Z, U)$}

Using Theorems 3.1 and 3.2 , we determine below the chromatic number of the distance graph $G(Z, U)$.

Theorem 4.1. Let $U=\left\{U_{r}, U_{r+1}, \ldots, U_{n}\right\}$. Then

(i) if $r+1 \leq n \leq 2 r+1$, then

$$
\frac{1}{\mu(U)}=\chi_{f}(G(Z, U))=\chi_{c}(G(Z, U))=\chi(G(Z, U))=\frac{1}{\kappa(U)}=3 ;
$$

(ii) if $n>2 r+1$, then

and

$$
3<\frac{1}{\mu(U)}=\chi_{f}(G(Z, U)) \leq \chi_{c}(G(Z, U)) \leq \frac{1}{\kappa(U)}<4,
$$

$$
\chi(G(Z, U))=4 .
$$

P r o o f. Using Theorems 1.1 and 1.2 for a distance set $D$, we have

and

$$
\frac{1}{\mu(D)}=\chi_{f}(G(Z, D)) \leq \chi_{c}(G(Z, D)) \leq \frac{1}{\kappa(D)},
$$

$$
\left\lceil\chi_{c}(G(Z, D))\right\rceil=\chi(G(Z, D)) \text {. }
$$

Therefore, by Theorem 3.1, if $r+1 \leq n \leq 2 r+1$, then

$$
\frac{1}{\mu(U)}=\chi_{f}(G(Z, U))=\chi_{c}(G(Z, U))=\chi(G(Z, U))=\frac{1}{\kappa(U)}=3 .
$$

Next, if $n>2 r+1$, then using Theorem 3.1 and Theorem 3.2.

and

$$
3<\frac{1}{\mu(U)}=\chi_{f}(G(Z, U)) \leq \chi_{c}(G(Z, U)) \leq \frac{1}{\kappa(U)}<4,
$$

$$
\chi(G(Z, U))=\left\lceil\chi_{c}(G(Z, U))\right\rceil=4 .
$$




\section{ANSHIKA SRIVASTAVA — RAM KRISHNA PANDEY — OM PRAKASH}

Acknowledgements 1. The authors are very much thankful to the referee for the careful reading and giving very useful comments. These comments were very useful to present the paper in a much better form. The authors are also thankful to Professor G e or g es Grek os for his useful suggestions besides the referee's remarks.

\section{REFERENCES}

[1] CANTOR, D. G.-GORDON, B.: Sequences of integers with missing differences, J. Combin. Theory Ser. A 14 (1973), 281-287.

[2] CHANG, G.-LIU, D. D.-F.-ZHU, X.: Distance graphs and T-colorings, J. Combin. Theory Ser. B 75 (1999), 159-169.

[3] CIPU, M.-LUCA, F.: On the Galois group of the generalized Fibonacci polynomial, An. Ştiinţ. Univ. Ovidius Constanţa Ser. Mat. 9 (2001), 27-38.

[4] COllister, D.-LIU, D. D.-F.: Study of $\kappa(D)$ for $D=\{2,3, x, y\}$, IWOCA (2014), 250-261.

[5] GRIGGS, J. R.-LIU, D. D.-F.: , The channel assignment problem for mutually adjacent sites, J. Combin. Theory Ser. A 68 (1994), 169-183.

[6] GUPTA, S.-TRIPATHI, A.: Density of M-sets in arithmetic progression Acta Arith. 89 (1999), 255-257.

[7] HARALAMBIS, N. M.: Sets of integers with missing differences, J. Combin. Theory Ser. A 23 (1977), 22-33.

[8] LIU, D. D.-F.-ZHU, X.: Fractional chromatic number for distance graphs with large clique size, J. Graph Theory 47 (2004), 129-146.

[9] Fractional chromatic number of distance graphs generated by two-interval sets, European J. Combin. 29 (7) (2008), 1733-1742.

[10] MILES, E. P., JR.: Generalized Fibonacci numbers and associated matrices, Amer. Math. Monthly 67 (1960), 745-752.

[11] PANDEY, R. K.-TRIPATHI, A.: A note on the density of M-sets in geometric sequence, Ars Comb. CXIX (2015), 221-224.

[12] _ A note on a problem of Motzkin regarding density of integral sets with missing differences, J. Integer Sequences 14 (2011).

[13] On the density of integral sets with missing differences from sets related to arithmetic progressions J. Number Theory 131 (2011), 634-647. 


\title{
MOTZKIN'S MAXIMAL DENSITY AND RELATED CHROMATIC NUMBERS
}

[14] WOLFRAM, D. A.: Solving generalized Fibonacci recurrences, Fibonacci Quart. 36 (1998), 129-145.

[15] ZHU, X.: Circular chromatic number: A survey, Disc. Math. 229 (2001), 371-410.

Received November 10, 2016

Accepted March 23, 2017

\author{
Anshika Srivastava \\ Department of Mathematics \\ Indian Institute of Technology Patna \\ Patna - 800013 \\ INDIA \\ E-mail: anshikasme@gmail.com

\section{Ram Krishna Pandey} \\ Department of Mathematics \\ Indian Institute of Technology Roorkee \\ Roorkee - 247667 \\ INDIA \\ E-mail: ramkpandey@gmail.com \\ Om Prakash \\ Department of Mathematics \\ Indian Institute of Technology Patna \\ Patna - 800013 \\ INDIA \\ E-mail: om@iitp.ac.in
}

